\title{
BMJ Open Study of potentially preventable hospitalisations (PPH) for chronic conditions: what proportion are preventable and what factors are associated with preventable PPH?
}

\author{
Jennifer Johnston (D) , ${ }^{1}$ Jo Longman, ${ }^{1}$ Dan Ewald, ${ }^{1}$ Jonathan King, ${ }^{2}$ Sumon Das, ${ }^{3}$ \\ Megan Passey ${ }^{1}$
}

To cite: Johnston J, Longman J, Ewald D, et al. Study of potentially preventable hospitalisations (PPH) for chronic conditions: what proportion are preventable and what factors are associated with preventable PPH? BMJ Open 2020;10:e038415. doi:10.1136/ bmjopen-2020-038415

- Prepublication history and additional materials for this paper is available online. To view these files, please visit the journal online (http://dx.doi. org/10.1136/bmjopen-2020038415).

Received 11 March 2020 Revised 08 0ctober 2020 Accepted 17 October 2020

Check for updates

(c) Author(s) (or their employer(s)) 2020. Re-use permitted under CC BY-NC. No commercial re-use. See rights and permissions. Published by BMJ.

${ }^{1}$ University Centre for Rural Health, University of Sydney, Lismore, New South Wales, Australia

${ }^{2}$ The Kirby Institute, Kensington, New South Wales, Australia ${ }^{3}$ Child Health Division, Menzies School of Health Research, Casuarina, Northern Territory, Australia

Correspondence to

Dr Megan Passey;

megan.passey@sydney.edu.au

\section{ABSTRACT}

Introduction The proportion of potentially preventable hospitalisations (PPH) which are actually preventable is unknown, and little is understood about the factors associated with individual preventable PPH. The Diagnosing Potentially Preventable Hospitalisations (DaPPHne) Study aimed to determine the proportion of PPH for chronic conditions which are preventable and identify factors associated with chronic PPH classified as preventable. Setting Three hospitals in NSW, Australia.

Participants Community-dwelling patients with unplanned hospital admissions between November 2014 and June 2017 for congestive heart failure (CHF), chronic obstructive pulmonary disease (COPD), diabetes complications or angina pectoris. Data were collected from patients, their general practitioners (GPs) and hospital records.

Outcome measures Assessments of the preventability of each admission by an Expert Panel.

Results 323 admissions were assessed for preventability: $46 \%(148 / 323)$ were assessed as preventable, $30 \%$ $(98 / 323)$ as not preventable and $24 \%(77 / 323)$ as unclassifiable. Statistically significant differences in proportions preventable were found between the three study sites $(29 \% ; 47 \% ; 58 \% ; p \leq 0.001)$ and by primary discharge diagnosis $(p \leq 0.001)$.

Significant predictors of an admission being classified as preventable were: study site; final principal diagnosis of CHF; fewer diagnoses on discharge; shorter hospital stay; GP diagnosis of COPD; GP consultation in the last 12 months; not having had a doctor help make the decision to go to hospital; not arriving by ambulance; patient living alone; having someone help with medications and requiring help with daily tasks.

Conclusions That less than half the chronic PPH were assessed as preventable, and the range of factors associated with preventability, including site and discharge diagnosis, are important considerations in the validity of PPH as an indicator. Opportunities for interventions to reduce chronic $\mathrm{PPH}$ include targeting patients with $\mathrm{CHF}$ and COPD, and the provision of social welfare and support services for patients living alone and those requiring help with daily tasks and medication management.

\section{Strengths and limitations of this study}

- Focusing on the four most common chronic potentially preventable hospitalisations principal discharge diagnoses enabled examination of this general patient group without confounding of factors associated with acute and vaccine-preventable admissions.

- Undertaking the study across multiple sites (including both metropolitan and regional hospitals) allowed for examination of geographic and site variability.

- The collection of comprehensive information from patients, their general practitioners (GPs) and hospital records facilitated consideration of patient, clinician and system factors in assessments of the preventability of individual admissions, as well as consideration of the broad range of factors potentially associated with admissions assessed as preventable.

- Only admissions with full data (ie, from patients, their GPs and hospital records) were assessed by the Expert Panel, with these patients found to be slightly healthier (ie, lower rates of multi-morbidity and lower levels of psychological distress) than those not assessed, potentially limiting the generalisability of the study findings.

- The smaller than anticipated sample reduced the power of the model and despite a comprehensive list of predictor variables, the model only explained $22 \%$ of the preventability of the admissions, suggesting even greater complexity in the drivers of preventability.

\section{INTRODUCTION}

Potentially preventable hospitalisations (PPH) are broadly defined as unplanned hospital admissions which could potentially have been prevented with effective, timely outpatient care prior to the admission. ${ }^{12}$ Admissions are classified as PPH according to their final discharge code, and are classed 
as chronic, acute or vaccine-preventable. In Australia, approximately half $(46 \%)$ of all $\mathrm{PPH}$ are classed as chronic, ${ }^{1}$ the majority of which are for congestive heart failure $(\mathrm{CHF})$, chronic obstructive pulmonary disease (COPD), diabetes complications or angina pectoris. ${ }^{3}$

Reducing rates of $\mathrm{PPH}$ are a priority internationally, ${ }^{4-7}$ although there is little evidence that efforts to do so have been successful. ${ }^{89}$

Although rates of $\mathrm{PPH}$ are widely used as a proxy measure or indicator of access to timely and effective primary care, ${ }^{10}$ the proportion of PPH which are actually preventable is unknown, ${ }^{11}$ and the validity of $\mathrm{PPH}$ as a marker for preventability has not been confirmed empirically. ${ }^{71213}$ As the classification of an admission as a PPH is based on diagnostic codes for a specific list of conditions at discharge rather than an assessment of preventability, admissions classified as PPH will include some admissions which are preventable and those which are not, ${ }^{6}$ and miss some admissions which are preventable.

Few studies have attempted to assess the preventability of individual admissions, and most work in this area has focused on readmissions. A systematic review of the preventability of readmissions, drawing on studies which used a range of non-comprehensive and widely varying methods for assessing preventability, reported estimates ranging from $5 \%$ to $79 \% .{ }^{14}$ Further, little is known about the factors associated with PPH which are actually preventable. The importance of identifying which patients are likely to benefit from interventions, that is, those which are 'impactible', ${ }^{15}$ and of learning from PPH that could have been prevented, ${ }^{5}$ is being increasingly recognised in the literature. To our knowledge, no previous research has been undertaken specifically examining the factors associated with preventable $\mathrm{PPH}{ }^{7}$

A wide range of factors have been found to be associated with rates and risk of chronic PPH more generally, irrespective of their actual preventability. Sociodemographic and health characteristics and behaviours have been found to be the major drivers of geographic variation in rates of $\mathrm{PPH}$ for chronic conditions in Australia. ${ }^{16}$ Previous research has shown that rates of chronic PPH are higher among men, ${ }^{17}$ in Aboriginal populations ${ }^{18}$ and rural areas, ${ }^{17}$ and those of older age, ${ }^{16}$ low socioeconomic status ${ }^{1719} 20$ and poorer health (self-reported and greater number of comorbidities). ${ }^{16172122}$ Not engaging in routine physical activity, ${ }^{22}$ poor health literacy ${ }^{23}$ and poor medication adherence ${ }^{24}$ are also associated with increased risk of chronic PPH . Social isolation has been identified as an important contributory factor to chronic $\mathrm{PPH},{ }^{25}$ and higher social isolation scores are associated with more frequent chronic PPH ${ }^{26}$

Clinician-level factors associated with increased risk of chronic PPH include having multiple providers, ${ }^{27}$ low levels of provider continuity ${ }^{2829}$ or having a general practitioner (GP) with fewer (less than 10) years of experience. ${ }^{17}$ Having a GP with less access to ancillary services, ${ }^{17}$ lower levels of collaboration with other community-based providers $^{28}$ or low adherence to clinical guidelines ${ }^{17}$ are also associated with higher risk for chronic PPH .

At the system level, the findings regarding access to care and risk of chronic PPH are mixed. Research undertaken in the USA found low self-reported access to care to be associated with higher rates of chronic PPH admissions, ${ }^{2}$ and a systematic review concluded that adequate physician supply is associated with reduced rates of chronic $\mathrm{PPH} .{ }^{28}$ However, Australian research found area level primary care providers supply to explain only $3.5 \%$ of the variation in chronic PPH rates, ${ }^{16}$ and better access to primary care providers has been found to be associated with higher rates of chronic PPH (although again only explaining a small proportion of the variation). ${ }^{20}$

Although a range of factors across patient, clinician and system levels have been found to be associated with risk for unplanned hospital admissions, some of the findings are equivocal and the role of such factors in PPH which are actually preventable is unknown, limiting our ability to develop and target appropriate interventions. To better understand and ultimately reduce rates of $\mathrm{PPH}$, a means of identifying and examining those $\mathrm{PPH}$ which are actually preventable is required.

The Diagnosing Potentially Preventable Hospitalisations (DaPPHne) Study ${ }^{30}$ aimed to (1) identify the proportion of $\mathrm{PPH}$ admissions for chronic conditions which are preventable and (2) to generate an evidence base identifying modifiable factors driving preventable PPH for chronic conditions. This paper addresses these central aims of the DaPPHne Study, by describing the DaPPHne participants, the proportion of admissions assessed as preventable and the factors associated with admissions assessed as preventable.

\section{METHOD}

The DaPPHne Study is a mixed methods data linkage study, described in detail in previous papers. ${ }^{30}{ }^{31}$ Patients with unplanned admissions to one metropolitan and two regional hospitals in NSW, Australia, with a primary discharge diagnosis of COPD, CHF, diabetes complications or angina pectoris; aged 45 years and over; community-dwelling; able to give informed consent and not transferred from another hospital, were eligible to participate in the study.

Patients were recruited to the study over recruitment periods of approximately 12 months at each site, between November 2014 and June 2017. Research nurses screened admissions to the emergency departments to identify patients who were potentially eligible to participate in the study. Research nurses monitored patients' progress and approached those who were admitted to hospital to invite them to participate in the study. As inclusion criteria included a primary discharge diagnosis of COPD, CHF, diabetes complications or angina pectoris, a final determination of if a patient was eligible to be included in the study could not be made until the admission had been coded following their discharge from hospital. 
Following the provision of written informed consent, data were collected via (a) patient questionnaire (during the hospital admission), (b) GP (ie, family physician) structured interview and (c) extraction of hospital clinical data. Please see online supplemental data collection tool. The patient questionnaire included demographic characteristics, health conditions and self-reported functioning, disease self-management, use of health services, health literacy, psychological distress and social support. GPs were interviewed face-to-face (the vast majority) or over the phone regarding care provided, including adherence to selected elements of the guidelines for management of patients' PPH diagnoses; the potential impact of other chronic conditions (including mental health) and any social issues relating to the admission and the patient's use of out-of-hospital clinical and non-clinical services. Clinical data were extracted from the hospital records for the participants' current admission and the most recent previous admission, including principal diagnoses and comorbidities, medications on admission and discharge information. Copies of the clinical notes for the period up to and including the first 24 hours after admission and the hospital discharge summary were also provided.

Assessment of the preventability of each admission was made by an Expert Panel. ${ }^{31}$ Expert Panels were established at each of the three sites, and consisted of a local hospital physician, GP and community nurse with expertise in chronic disease management, ensuring a diverse range of perspectives on patient care, as well as clinical expertise and knowledge of local services and systems. Expert Panel members each reviewed a detailed case report of each admission (containing information from the patient questionnaire, GP interview, hospital data, 24 hours of clinical notes and discharge summary) and independently assessed if they were reasonably confident the admission was preventable (yes/no) within the framework of the study definition (see below). For those admissions where there was not consensus on this question, panel members met for a facilitated discussion about the admission to reach consensus. The panel members were also asked to assess whether they were reasonably confident the admission was not preventable. For those admissions classified as 'preventable', Expert Panel members identified intervention/s they considered could have prevented the admission.

\section{Definition of preventable admissions}

The DaPPHne Study defines 'preventable' admissions as unplanned admissions which could have been prevented if: (1) appropriate, adequate, accessible and good quality support in the community had been available and accessed in the preceding 3 months (support in the community might include primary healthcare, family/ neighbour/friend/social support, health or non-health community services); and/or (2) appropriate individual health behaviours, for example, disease self-management, had occurred in the 3 months prior to admission.
Although the original work of Billings and colleagues ${ }^{32}$ referred to the 'period immediately prior to admission', we were unable to identify any clear timeframes for preventability in the literature. In consultation with clinicians, it was determined that a period of 3 months prior to the admission to be a reasonable time in which to expect any interventions or behaviour change aimed at preventing admission to be effective. ${ }^{12} 31$

\section{Patient and public involvement}

A priority of the DaPPHne Study was to better understand patients' experiences regarding their unplanned hospital admission and the management of their chronic condition/s. This was identified by the research team as both a gap in the literature, and information vital for the development of interventions designed to reduce chronic PPH and contribute to improvements in clinical care and self-management. Membership of the DaPPHne Steering Committee included a consumer representative, who provided input and guidance regarding the study design (including a substudy specifically exploring patient perspectives $^{33}$ ) and implementation, and the development of the patient questionnaire. Patients were not involved in the recruitment to, nor conduct of the study, other than as participants. A plain language summary of the main findings has been disseminated to those participants who indicated during the informed consent process that they would like to receive a summary of the findings.

\section{Analysis}

Data were entered into a specifically designed Access database, with all analyses completed using Stata V.15. ${ }^{34}$ Descriptive statistics of the characteristics of the patients and their admissions were generated.

The sample was divided into two groups: those assessed as 'preventable' and those assessed as 'other' (consisting of admissions assessed as not preventable and those admissions not able to be classified as preventable or not preventable). This approach was consistent with the research aims of assessing the extent of preventability of chronic PPH and the factors associated with preventable chronic PPH .30 The 'preventable' and 'other' groups were compared on all variables collected, using $\chi^{2}$, Fisher's exact, t-tests or non-parametric equivalents, as appropriate.

\section{Multivariable analysis}

Initial bivariate significance testing was conducted to identify potentially important model candidates for inclusion in a multivariable analysis. Those characteristics that demonstrated a $p$ value of 0.2 or lower were selected as potential candidates. Consistent with recommendations by Kahan and colleagues, we accounted for the impact of site on the outcomes by including hospital site as a fixedeffect in the logistic regression modelling. This is recommended for situations where there are a small number of clusters. ${ }^{35}$ Age, sex and Indigenous status were included as candidates regardless of their statistical significance. 
The multivariable logistic regression model construction process was based on the methods described by Bursac and colleagues. ${ }^{36}$ Each candidate variable was then included in the model one at a time with the candidate variable demonstrating the smallest $p$ value then added to the model. This was repeated in a stepwise manner with the remaining candidate variables until there were no more variables demonstrating $\mathrm{p}$ values of 0.1 .

If a candidate variable was added which then made another variable already in the model go over the 0.1 threshold, the latter was then removed from the model and changes in Akaike information criterion (AIC), goodness of fit and area under the receiver operating characteristics (ROC) curve were analysed. If considerable changes to these measures were made $(\geq 15 \%)$, both variables were retained. If the changes were not considerable, the variable which was over the threshold was removed from the model and returned to the group of candidate variables further considered for inclusion in later models.

After there were no variables left demonstrating a $p$ value of 0.1 or less the rest of the candidate variables were then added one at a time with each model tested for considerable $(\geq 15 \%)$ changes in AIC, goodness of fit, the area under the ROC and confounding of the effect sizes of the rest of the included variables. Any variables that altered the model under these criteria were included in the model. Finally, all included variables were tested for interactions with hospital site. Any significant interactions were retained in the model.

To assess potential selection bias, the admissions assessed by the Expert Panel (ie, those with data from all three sources: patient questionnaire, GP interview and hospital records) were compared with those recruited and eligible, but with no GP data, on all variables collected in the patient questionnaire and the hospital clinical audit (due to GP refusal or unable to identify or contact).
RESULTS

The numbers of patients who were screened, recruited and remained in the study are presented in figure 1 . Of the 1808 admissions deemed potentially eligible after initial screening, 500 were 'missed'. In these cases, patients were discharged after a very short admission, commonly during periods when the research nurses were not working (ie, weekends or out of business hours during the week), and as such, there was not an opportunity to recruit them to the study.

Of 791 patients consenting to participate, 545 were determined as eligible (following the assignment of final discharge diagnoses). Full data were collected for 323 eligible admissions and were assessed by the Expert Panels. It was not possible to undertake GP interviews for 222 of the eligible admissions and as such, these were not assessed by the Expert Panels.

A comparison of admissions assessed by the Expert Panels $(n=323)$ and those not assessed $(n=222)$, on all variables collected in the patient questionnaire and the hospital clinical audit found a small number of significant differences between the groups. Those assessed by the Expert Panel were less likely to have consulted their GP about the condition for which they were admitted to hospital within the past 3 months (78\% of those assessed vs $86 \%$ of those not, $\mathrm{p}=0.016$ ); more likely to have very good health literacy on the Partners in Health Scale ${ }^{37}$ ( $57 \%$ vs $46 \%, \mathrm{p}=0.009$ ) and ninth grade or above literacy on the Rapid Estimate of Adult Literacy in Medicine $\left(\right.$ REALM-R) ${ }^{38}$ ( $73 \%$ vs $58 \%, \mathrm{p}<0.001$ ); less likely to have moderate or severe psychological distress (as assessed by the Kessler Psychological Distress Scale (K10) ${ }^{39}$ (34\% vs $44 \%, \mathrm{p}=0.022$ ); more likely to eat at least two servings of fruit/day ( $52 \%$ vs $41 \%, \mathrm{p}=0.012)$; more likely to have a note in their hospital records that they were having difficulty managing at home $(20 \%$ vs $9 \%, \mathrm{p}<0.001)$ and less likely to have seven or more diagnoses on admission $(39 \%$

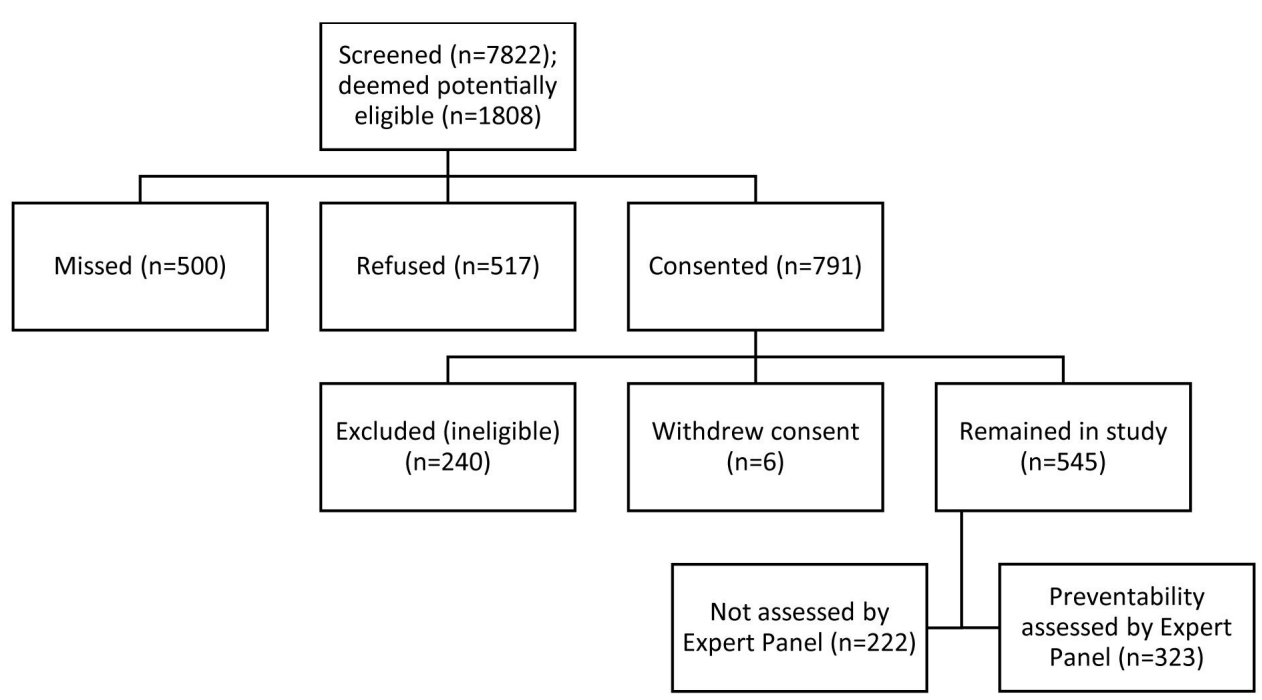

Figure 1 Screening, recruitment and eligibility of DaPPHne participants across the three study sites. DaPPHne, Diagnosing Potentially Preventable Hospitalisations. 
vs $47 \%, \mathrm{p}=0.046)$. There were no differences on final diagnoses (principal or total number), number of prescribed medications or other health risk behaviours. Given the lower rate of multi-morbidity, better health literacy and lower psychological distress among those assessed, those assessed by Expert Panels may be slightly healthier than those not assessed by Expert Panels.

\section{Patient characteristics}

A detailed description of the 323 assessed by the Expert Panel admissions is provided in online supplemental table S1. Average age was 71 years (range 46-91 years). Just over half $(54 \%)$ were men and most were born in Australia $(71 \%)$, with only $14(4 \%)$ participants identifying as Aboriginal. In general, self-reported physical and mental health was poor: Approximately half (53\%) rated their general health as fair or poor and one-third $(34 \%)$ reported experiencing moderate to high psychological distress. Over half (55\%) were considered by their GP to have social issues impacting their ability to manage their health. Three-quarters (74\%) reported that they did not engage in any moderate physical activity. Of the 218 participants with body mass index calculated, $73 \%$ were classified as obese.

Most (78\%) participants reported having consulted a GP about their (primary) chronic condition in the previous 3 months. The majority $(82 \%)$ reported having more than one doctor (GPs or specialists) involved in their care, and many had also consulted other health professionals in the previous 12 months.

The most common principal diagnosis on discharge was COPD (38\%), followed by diabetes and/or related complications (27\%), CHF (25\%) and angina pectoris $(10 \%)$. There was considerable multi-morbidity, with $48 \%$ of admissions having seven or more diagnoses on discharge (ie, comorbidities that required ongoing management). Polypharmacy was also common, with $79 \%$ of the participants prescribed five or more prescription medications on admission.

\section{Proportion of admissions assessed as preventable}

Of the 323 admissions assessed, Expert Panel members agreed regarding preventability in $170(53 \%)$ of cases following their initial, individual assessments. The remaining cases required a facilitated discussion to reach consensus.

The Expert Panels assessed $148(46 \%)$ of the 323 admissions as preventable ('reasonably confident that this admission was preventable'). They were 'reasonably confident' that $98(30 \%)$ admissions were not preventable, with the remaining $77(24 \%)$ not able to be classified as preventable or not preventable. There were significant differences between the proportion of admissions assessed as preventable at the three study sites (29\%; $47 \% ; 58 \% ; \mathrm{p} \leq 0.001)$, and by principle discharge diagnosis, with CHF more likely to be assessed as preventable $(\mathrm{p} \leq 0.001)($ table 1$)$.
Table 1 Panel assessment of preventability by site and final principal discharge diagnosis

\begin{tabular}{|ccccc}
\hline & $\begin{array}{l}\text { Preventable } \\
(\%)\end{array}$ & $\begin{array}{l}\text { Other } \\
(\%)\end{array}$ & $\begin{array}{l}\text { Row total } \\
(\%)\end{array}$ & P value \\
\hline Panel assessment by site & & & \\
\hline Rural 1 & $25(29)$ & $62(71)$ & 87 & \\
Rural 2 & $58(47)$ & $65(53)$ & 123 & \\
Metro & $65(58)$ & $48(42)$ & 113 & \\
\hline Total & $148(46)$ & $175(54)$ & 323 & $<0.001$ \\
\hline Panel assessment by final principal discharge diagnosis & \\
\hline CHF & $50(63)$ & $29(37)$ & 79 & \\
\hline COPD & $58(47)$ & $65(53)$ & 123 & \\
Angina & $9(27)$ & $24(73)$ & 33 & $<0.001$ \\
\hline Diabetes & $31(35)$ & $57(65)$ & 88 & \\
\hline Total & $148(46)$ & $175(54)$ & 323 & \\
\hline
\end{tabular}

CHF, congestive heart failure; COPD, chronic obstructive pulmonary disease.

\section{How did the preventable admissions differ to the others?}

Statistically significant differences were found between the 'preventable' $(n=148)$ and 'other' $(n=175)$ admissions on a range of patient, clinician and system level (see online supplemental table S1). In univariate analyses, patients who were married or in a de facto relationship were less likely to have a preventable admission $(\mathrm{p}=0.028)$. Patients who were living alone $(\mathrm{p}=0.012)$, and those who regularly requiring help with daily tasks $(\mathrm{p}=0.023)$ were more likely to have a preventable admission. Patients with GPs reporting that they were aware of social issues that impacted negatively on the patients' ability to manage their health were more likely to have an admission assessed as preventable $(\mathrm{p}=0.018)$. Patients who did not engage in at least one 30 min session of moderate physical activity per week were also were also more likely to have an admission assessed as preventable $(\mathrm{p}=0.026)$.

Admissions with a final principal discharge diagnosis of CHF were more likely to be assessed as preventable $(\mathrm{p}<0.001)$. Indeed, patients with any diagnosis (ie, primary or secondary) of CHF ( $\mathrm{p}=0.016$ ) were more likely to be assessed as having had a preventable admission, as were those with any diagnosis of COPD $(p=0.040)$. In contrast, those patients with a principle discharge diagnosis of diabetes $(\mathrm{p}=0.019)$ or angina pectoris $(\mathrm{p}=0.024)$ were significantly less likely to be assessed as having had a preventable admission. Extent of comorbidity was also associated with preventability, with patients with one or two diagnoses on admission $(\mathrm{p}=0.028)$ and discharge $(\mathrm{p}=0.001)$ more likely to be assessed as having had a preventable admission. Those patients arriving by ambulance were less likely to be assessed as preventable $(\mathrm{p}=0.040)$.

\section{Predictors of preventability}

A multivariate model was built, with the final model shown in table 2. After controlling for age, sex and Indigenous 
Table 2 Multivariable logistic regression model of the significant predictors of PPH

\begin{tabular}{|c|c|c|c|c|}
\hline Predictor & OR & $P$ value & $95 \% \mathrm{Cls}$ & $90 \% \mathrm{Cls}$ \\
\hline \multicolumn{5}{|l|}{ Hospital site } \\
\hline Rural 1 & 1 & - & - & - \\
\hline Rural 2 & 3.98 & $<0.001$ & 1.9 to 8.31 & 2.14 to 7.38 \\
\hline Metro & 6.31 & $<0.001$ & 2.94 to 13.53 & 3.33 to 11.97 \\
\hline \multicolumn{5}{|l|}{ Final principal diagnosis of $\mathrm{CHF}$} \\
\hline No & 1 & - & - & - \\
\hline Yes & 5.43 & $<0.001$ & 2.71 to 10.89 & 3.03 to 9.74 \\
\hline \multicolumn{5}{|l|}{ Total diagnoses on discharge } \\
\hline 1-2 diagnoses & 1 & - & - & - \\
\hline Three and above diagnoses & 0.21 & 0.007 & 0.07 to 0.65 & 0.08 to 0.54 \\
\hline \multicolumn{5}{|l|}{ Length of hospital stay (days) } \\
\hline $1-2$ days & 1 & - & - & - \\
\hline Three days and above & 0.54 & 0.067 & 0.28 to 1.04 & 0.31 to 0.94 \\
\hline \multicolumn{5}{|l|}{ Mode of arrival to hospital } \\
\hline Ambulance & 1 & - & - & - \\
\hline Other & 1.75 & 0.055 & 0.99 to 3.1 & 1.08 to 2.82 \\
\hline \multicolumn{5}{|c|}{ A doctor helped make decision to go to hospital } \\
\hline No & 1 & - & - & - \\
\hline Yes & 0.39 & 0.01 & 0.19 to 0.8 & 0.22 to 0.71 \\
\hline
\end{tabular}

Any GP consult in the last 12 months

\begin{tabular}{|c|c|c|c|c|}
\hline No & 1 & - & - & - \\
\hline Yes & 3.48 & 0.03 & 1.13 to 10.8 & 1.36 to 9.02 \\
\hline \multicolumn{5}{|c|}{ GP diagnosis of COPD } \\
\hline No & 1 & - & - & - \\
\hline Yes & 1.64 & 0.093 & 0.92 to 2.90 & 1.01 to 2.65 \\
\hline \multicolumn{5}{|l|}{ Living alone } \\
\hline Not alone & 1 & - & - & - \\
\hline Alone & 2.77 & 0.001 & 1.49 to 5.17 & 1.64 to 4.68 \\
\hline \multicolumn{5}{|c|}{ Patient has someone that helps with taking medications } \\
\hline No & 1 & - & - & - \\
\hline Yes & 2.35 & 0.028 & 1.1 to 5.04 & 1.24 to 4.46 \\
\hline \multicolumn{5}{|c|}{ Participant requires help with daily tasks } \\
\hline No & 1 & - & - & - \\
\hline Yes & 1.74 & 0.066 & 0.96 to 3.15 & 1.06 to 2.86 \\
\hline Constant & 0.126089 & 0.028 & 0.019829 & 0.801792 \\
\hline
\end{tabular}

Pseudo $R^{2}=0.2194$

Goodness of fit $p$ value $=0.5649$

Model adjusted for age, sex and Indigenous status.

$\mathrm{CHF}$, congestive heart failure; COPD, chronic obstructive pulmonary disease; GP, general practitioner; PPH, potentially preventable hospitalisations.

status, 11 variables were important predictors of an admission being classified as preventable (table 2).

At the patient level, predictors of an admission being classified as preventable were the patient living alone, having someone that helps with their medication, and requiring regular help with daily tasks. Having a final principle diagnosis of CHF, two or less diagnoses on discharge and having been diagnosed by their GP as having COPD were also predictors of an admission being classified as preventable. Other predictors related to having had a doctor help make the decision to go to hospital (less likely to be preventable) and having had a 
GP consultation in the previous 12 months (more likely to be preventable). Length of hospital stay of one to 2 days and not arriving by ambulance were also predictors of admissions being assessed as preventable.

\section{DISCUSSION}

This is the first study to empirically assess the preventability of individual PPH for chronic conditions. Utilising Expert Panels to assess the preventability of each admission, the findings show nearly half of the chronic PPH were classified as preventable. Importantly, the findings also show that in nearly one-third of the cases assessed, the Expert Panels were reasonably confident the admission was not preventable, that is, the panel deemed that there was nothing that could have been done in the 3 months leading up to the admission to prevent it. Together, these findings provide the first empirical evidence of the extent to which rates of PPH for chronic conditions overestimate the proportion of admissions which are actually preventable. Furthermore, the significant variability in the proportion of admissions assessed as preventable by both site and final discharge diagnosis has considerable implications for understanding what PPH is, and is not, measuring as a health system performance measure. These findings confirm the 'bluntness' of the measure,$^{40}$ and point to some of the limitations of this approach to inform policy and practice. ${ }^{12}$ The difference by site in the rates of PPH deemed preventable emphasises the importance of considering the factors, for instance service availability and accessibility, which contribute to preventable PPH at the local level. A forthcoming paper from the DaPPHne Study describing a qualitative analysis of clinicians' (including Expert Panel members) perspectives of what could have been done to prevent the hospitalisations assessed as preventable, examines the site-specific factors in detail (Longman et al, What could prevent chronic condition admissions assessed as preventable in rural and metropolitan contexts? Clinicians' perspectives from the DaPPHne study). Similarly, the difference by principle diagnosis in the rates of $\mathrm{PPH}$ deemed preventable emphasises the importance of considering the factors which contribute to preventable PPH by diagnosis.

It has previously been noted that the reliance on rates of PPH as an indicator is 'driven by the lack of alternative sources of data which capture the impact of ambulatory care' (p. 432). ${ }^{5}$ The DaPPHne Study includes a data linkage component which will link DaPPHne data to routinely collected administrative data (emergency department presentations, hospital admissions and mortality) with the aim of building predictive models to provide insights into refining the PPH indicator. ${ }^{30}$

The DaPPHne participants represent a group of patients, most of whom have complex needs, with limited social and financial resources, multi-morbidity, polypharmacy and poor health risk profiles. For many, their existing health conditions are difficult to manage, highlighting the importance of prevention at a much earlier stage. Nevertheless, the findings presented here point to possible leverage points in the 3 months leading up to an admission, using interventions designed to foster better clinical care and self-management in the community. The findings indicate that, of the four chronic diseases included in our study, the two which particularly represent opportunities for action are CHF and COPD. CHF as principal discharge diagnosis was a significant predictor of being assessed as preventable in the multivariate analysis, with $63 \%$ of admissions with $\mathrm{CHF}$ as principal discharge diagnosis assessed as preventable. As such, admissions with $\mathrm{CHF}$ as principle discharge diagnosis accounted for $34 \%$ of all the preventable admissions in the study. In contrast, while a smaller proportion $(39 \%)$ of COPD admissions were preventable, these admissions accounted for a higher proportion (41\%) of the total preventable admissions in the study, due to the high number of COPD admissions. Given these findings, further examination of interventions targeting patients with these diagnoses are warranted. For example, the use of patient-tailored COPD action plans, ${ }^{41-43}$ as well as education interventions, ${ }^{44}$ show promise for improving outcomes and preventing admissions for patients with COPD. The findings regarding pulmonary rehabilitation are mixed and require further research to examine the nuances of such programmes and their impact on hospital admissions and other outcomes. ${ }^{45}$ Special clinics for heart failure have been found to reduce risk of unplanned admissions and readmissions, ${ }^{46}$ and a post-discharge intervention based on patient and family collaborations (including lifestyle modification) and designed according to patients' needs, has been found to be effective in reducing readmissions in CHF patients. ${ }^{47}$

Admissions for patients with less than three diagnoses were more likely to be assessed as preventable. In comparison to those patients with three or more diagnoses, these patients may represent a group with somewhat less complex needs and more scope for interventions to be of benefit. Similarly, shorter admissions (less than 3 days) were more likely to be assessed as preventable, again possibly reflecting less complex or clinically challenging scenarios which may have been managed in the community.

Patients' living arrangements, specifically living alone, was found to be a significant predictor of an admission being classified as preventable. One in five of the DaPPHne cohort reported having no or little social support, and previous work has highlighted the role that social isolation can have in unplanned admissions for chronic conditions. ${ }^{25}$ Other significant predictors of an admission being classified as preventable included the patient having someone who regularly helps them with their medication (eg, know when and/or what to take) and the patient needing help with daily tasks. These apparently conflicting results highlight the difficulty in establishing valid measures of important preventability factors. In this case it is likely having someone assist with medication was a marker of patient need rather than a cause of unplanned admission. A focus on social welfare 
and support services, including care in the home encompassing medication management and daily tasks, may enable patients to better manage their chronic conditions in the community. The forthcoming paper from the DaPPHne Study will further contribute to our understanding of possible leverage points by describing a qualitative analysis of clinicians' perspectives of what could have been done to prevent the hospitalisations assessed as preventable (Longman et al, What could prevent chronic condition admissions assessed as preventable in rural and metropolitan contexts? Clinicians' perspectives from the DaPPHne study).

Strengths of the DaPPHne Study included that, by focusing on the four most common chronic $\mathrm{PPH}$ principal discharge diagnoses, we could examine this general patient group and their PPH without the impact of confounding of factors associated with acute and vaccine-preventable admissions. Further, the inclusion of both metropolitan and regional sites strengthened the study by allowing for consideration of geographic variability. Comprehensive information from patient, GP and hospital records allowed for consideration of patient, clinician and system factors in both assessments of preventability of individual admissions, as well as examination of the factors associated with admissions assessed as preventable. The recruitment of Expert Panels for each of the three sites ensured their ability to make informed assessments of preventability within the context of local service systems. Furthermore, the consistencies across the three panels in terms of processes, training, tools and frameworks within which to make their assessments reduced the impact of difference between the approaches (and therefore assessments) of the Panels. ${ }^{31}$ It is possible, however, that some level of systematic differences between the panels may, nevertheless, have contributed to the site difference in the proportions assessed as preventable.

Limitations of the study included that the preventability of the eligible admissions with full data were assessed, thereby excluding those without GP interview. Given the lower rate of multi-morbidity, better health literacy and lower psychological distress among those assessed, those assessed by Expert Panel may be slightly healthier than those not assessed by Expert Panels, and therefore possibly more likely to have their admission assessed as preventable. It is also worth noting that a considerable number $(\mathrm{n}=500)$ of admissions considered 'potentially eligible' (following initial screening) were 'missed' by the Research Nurses, commonly due to short admissions over weekends. It is plausible that this may have also contributed to an underestimation of the proportion of preventable admissions, given that admissions of less than 3 days were more likely to be assessed as preventable.

Further, we anticipated recruiting a larger sample and greater participation by patients' GPs. The smaller than anticipated sample reduced the power of the model. Despite a comprehensive list of predictor variables, the model only explained $22 \%$ of the preventability of the admissions, suggesting even greater complexity in the drivers of preventability. Further research may aim to identify additional variables that we did not consider or measure variables we included in a different way.

As the first study to empirically assess the preventability of individual PPH for chronic conditions, DaPPHne makes an important contribution to the field. That less than half the PPH were assessed as preventable (and nearly one third as not preventable), and the wide range of factors associated with preventability, including site and discharge diagnosis, are important factors in future considerations in the validity and use of PPH as an indicator. Furthermore, the findings presented here underscore the importance of identifying which PPH are preventable, and the modifiable factors driving them. The improved understanding of which chronic PPH were preventable provided by this work points to opportunities for interventions to reduce PPH among people with $\mathrm{CHF}$ and $\mathrm{COPD}$, and the importance of the provision of social welfare and support services for patients living alone and those requiring help with daily tasks and medication management.

Contributors JL, DE and MP conceived and designed the study; JJ, JL, DE and MP all contributed to data collection. All authors (including JK and SD) contributed to the data analysis and interpretation of the findings, and to the drafting and revising of the paper.

Funding The DaPPHne Study was funded by the partner organisations: Western Sydney Local Health District, Mid North Coast Local Health District, North Coast Primary Health Network, New South Wales Agency for Clinical Innovation and University Centre for Rural Health, University of Sydney. Award/Grant number is not applicable.

Competing interests None declared.

Patient consent for publication Not required.

Ethics approval Ethical approval for the conduct of this study was granted by the New South Wales Population and Health Services Research Ethics Committee (ref HREC/14/CIPHS/39).

Provenance and peer review Not commissioned; externally peer reviewed.

Data availability statement Data are available upon reasonable request. Data are available from the corresponding author upon reasonable request.

Supplemental material This content has been supplied by the author(s). It has not been vetted by BMJ Publishing Group Limited (BMJ) and may not have been peer-reviewed. Any opinions or recommendations discussed are solely those of the author(s) and are not endorsed by BMJ. BMJ disclaims all liability and responsibility arising from any reliance placed on the content. Where the content includes any translated material, BMJ does not warrant the accuracy and reliability of the translations (including but not limited to local regulations, clinical guidelines, terminology, drug names and drug dosages), and is not responsible for any error and/or omissions arising from translation and adaptation or otherwise.

Open access This is an open access article distributed in accordance with the Creative Commons Attribution Non Commercial (CC BY-NC 4.0) license, which permits others to distribute, remix, adapt, build upon this work non-commercially, and license their derivative works on different terms, provided the original work is properly cited, appropriate credit is given, any changes made indicated, and the use is non-commercial. See: http://creativecommons.org/licenses/by-nc/4.0/.

\section{ORCID iD}

Jennifer Johnston http://orcid.org/0000-0001-6564-0728

\section{REFERENCES}

1 Australian Institute of Health and Welfare. Admitted patient care 2017-18: Australian hospital statistics in health services series. Canberra: AlHW, 2019.

2 Bindman AB, Grumbach K, Osmond D, et al. Preventable hospitalizations and access to health care. JAMA 1995;274:305-11. 
3 Australian Institute of Health and Welfare. Australian hospital statistics 2012-13. health services series No. 50. cat. No. HSE 145, 2014.

4 Busby J, Purdy S, Hollingworth W. A systematic review of the magnitude and cause of geographic variation in unplanned hospital admission rates and length of stay for ambulatory care sensitive conditions. BMC Health Serv Res 2015;15:324.

5 Hodgson K, Deeny SR, Steventon A. Ambulatory care-sensitive conditions: their potential uses and limitations. BMJ Publishing Group Ltd, 2019.

6 Steventon A, Deeny S, Friebel R, et al. Briefing: emergency hospital admissions in England: which may be avoidable and how? 2018.

7 Solberg LI. Preventable hospital admissions: are they? Fam Pract 2015;32:245-6.

8 Parry W, Wolters AT, Brine RJ, et al. Effect of an integrated care pathway on use of primary and secondary healthcare by patients at high risk of emergency inpatient admission: a matched control cohort study in tower Hamlets. BMJ Open 2019;9:e026470.

9 Plant NA, Kelly PJ, Leeder SR, et al. Coordinated care versus standard care in hospital admissions of people with chronic illness: a randomised controlled trial. Med J Aust 2015;203:33-8.

10 Australian Institute of Health and Welfare. Potentially preventable hospitalisations in Australia by age groups and small geographic areas, 2017-18. Cat. no: HPF 36:[PPH by PHN and SA3]. Available: https://www.aihw.gov.au/reports/primary-health-care/potentiallypreventable-hospitalisations/contents/overview [Accessed 9 Jan 2020].

11 Purdey S, Huntley A. Predicting and preventing avoidable hospital admissions: a review. J R Coll Physicians Edinb 2013;43:340-4.

12 Longman JM, Passey ME, Ewald DP, et al. Admissions for chronic ambulatory care sensitive conditions - a useful measure of potentially preventable admission? BMC Health Serv Res 2015;15:472.

13 Paul MCet al. Admissions for ambulatory care sensitive conditions: a national observational study in the general and COPD population. Eur J Public Health 2018.

14 van Walraven C, Bennett C, Jennings A, et al. Proportion of hospital readmissions deemed avoidable: a systematic review. CMAJ 2011;183:E391-402.

15 Steventon A, Billings J. Preventing hospital readmissions: the importance of considering 'impactibility, 'not just predicted risk. BMJ Publishing Group Ltd, 2017.

16 Falster MO, Jorm LR, Douglas KA, et al. Sociodemographic and health characteristics, rather than primary care supply, are major drivers of geographic variation in preventable hospitalizations in Australia. Med Care 2015;53:436-45.

17 O'Malley AS, Pham HH, Schrag D, et al. Potentially avoidable hospitalizations for COPD and pneumonia: the role of physician and practice characteristics. Med Care 2007;45:562-70.

18 Li SQ, Gray NJ, Guthridge SL, et al. Avoidable hospitalisation in Aboriginal and non-Aboriginal people in the Northern Territory. Med $J$ Aust 2009;190:532-6.

19 Page ACet al. Atlas of avoidable hospitalisations in Australia: ambulatory care-sensitive conditions. Adelaide: Public Health Information Development Unit, University of Adelaide, 2007.

20 Mazumdar Set al. Spatial clusters of chronic preventable hospitalizations (ambulatory sensitive conditions) and access to primary care. J Public Health 2019.

21 Payne RA, Abel GA, Guthrie B, et al. The effect of physical multimorbidity, mental health conditions and socioeconomic deprivation on unplanned admissions to hospital: a retrospective cohort study. CMAJ 2013;185:E221-8.

22 Bahadori K, FitzGerald JM. Risk factors of hospitalization and readmission of patients with COPD exacerbation-systematic review. Int J Chron Obstruct Pulmon Dis 2007;2:241-51.

23 Baker DW, Gazmararian JA, Williams MV, et al. Functional health literacy and the risk of hospital admission among Medicare managed care enrollees. Am J Public Health 2002;92:1278-83.

24 Vestbo J, Anderson JA, Calverley PMA, et al. Adherence to inhaled therapy, mortality and hospital admission in COPD. Thorax 2009;64:939-43.
25 Longman J, Passey M, Singer J, et al. The role of social isolation in frequent and/or avoidable hospitalisation: rural community-based service providers' perspectives. Aust Health Rev 2013;37:223-31.

26 Longman JM, I Rolfe M, Passey MD, et al. Frequent hospital admission of older people with chronic disease: a cross-sectional survey with telephone follow-up and data linkage. BMC Health Serv Res 2012;12:373.

27 Caughey GE, Pratt NL, Barratt JD, et al. Understanding 30-day re-admission after hospitalisation of older patients for diabetes: identifying those at greatest risk. Med J Aust 2017;206:170-5.

28 van Loenen T, van den Berg MJ, Westert GP, et al. Organizational aspects of primary care related to avoidable hospitalization: a systematic review. Fam Pract 2014;31:502-16.

29 Gill JM, Mainous AG. The role of provider continuity in preventing hospitalizations. Arch Fam Med 1998:7:352-7.

30 Passey ME, Longman JM, Johnston JJ, et al. Diagnosing potentially preventable hospitalisations (DaPPHne): protocol for a mixedmethods data-linkage study. BMJ Open 2015;5:e009879.

31 Johnston JJ, Longman JM, Ewald DP, et al. Validity of a too designed to assess the preventability of potentially preventable hospitalizations for chronic conditions. Fam Pract 2020;37:390-4

32 Billings J, Zeitel L, Lukomnik J, et al. Impact of socioeconomic status on hospital use in New York City. Health Aff 1993;12:162-73.

33 Longman JM, Rix E, Johnston JJ, et al. Ambulatory care sensitive chronic conditions: what can we learn from patients about the role of primary health care in preventing admissions? Aust J Prim Health 2018;24:304-10.

34 StataCorp. Stata statistical software: release 15. College Station, TX: StataCorp LLC, 2017.

35 Kahan BC. Accounting for centre-effects in multicentre trials with a binary outcome - when, why, and how? BMC Med Res Methodol 2014;14:20.

36 Bursac Z, Gauss CH, Williams DK, et al. Purposeful selection of variables in logistic regression. Source Code Biol Med 2008;3:17.

37 W Battersby M, Ask A, M Reece M, et al. The partners in health scale: the development and psychometric properties of a generic assessment scale for chronic condition self-management. Aust $J$ Prim Health 2003;9:41-52.

38 Bass PF, Wilson JF, Griffith CH. A shortened instrument for literacy screening. J Gen Intern Med 2003;18:1036-8.

39 Kessler RC, Barker PR, Colpe LJ, et al. Screening for serious mental illness in the general population. Arch Gen Psychiatry 2003;60:184-9.

40 Longman JM, Passey ME, Ewald DP. Sharper tools - chronic conditions and avoidable admission. Med J Aust 2013;199:395-6.

41 Lenferink A, van der Palen J, van der Valk PDLPM, et al. Exacerbation action plans for patients with COPD and comorbidities: a randomised controlled trial. Eur Respir J 2019;54:1802134.

42 Lenferink A, Brusse-Keizer M, van der Valk PD, et al. Selfmanagement interventions including action plans for exacerbations versus usual care in patients with chronic obstructive pulmonary disease. Cochrane Database Syst Rev 2017;8:CD011682.

43 Howcroft M, Walters EH, Wood-Baker R, et al. Action plans with brief patient education for exacerbations in chronic obstructive pulmonary disease. Cochrane Database Syst Rev 2016;12:CD005074

44 Murphy LA, Harrington P, Taylor SJ, et al. Clinical-effectiveness of self-management interventions in chronic obstructive pulmonary disease: an overview of reviews. Chron Respir Dis 2017;14:276-88.

45 Puhan MA, Gimeno-Santos E, Cates CJ, et al. Pulmonary rehabilitation following exacerbations of chronic obstructive pulmonary disease. Cochrane Database Syst Rev 2016;12:CD005305.

46 Thomas R, Huntley A, Mann M, et al. Specialist clinics for reducing emergency admissions in patients with heart failure: a systematic review and meta-analysis of randomised controlled trials. Heart 2013;99: :233-9.

47 Hasanpour-Dehkordi A, Khaledi-Far A, Khaledi-Far B, et al. The effect of family training and support on the quality of life and cost of hospital readmissions in congestive heart failure patients in Iran. Appl Nurs Res 2016;31:165-9. 